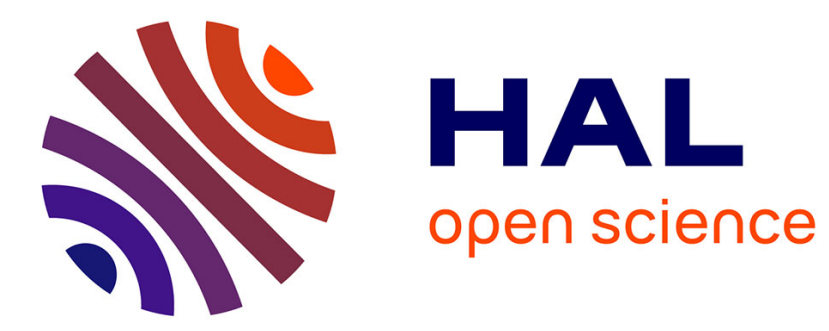

\title{
Les grandeurs de référence utilisables dans l'étude de l'alimentation en eau des cultures
}

\author{
Nader Katerji, Marc Hallaire
}

\section{To cite this version:}

Nader Katerji, Marc Hallaire. Les grandeurs de référence utilisables dans l'étude de l'alimentation en eau des cultures. Agronomie, 1984, 4 (10), pp.999-1008. hal-00884604

\section{HAL Id: hal-00884604 \\ https://hal.science/hal-00884604}

Submitted on 1 Jan 1984

HAL is a multi-disciplinary open access archive for the deposit and dissemination of scientific research documents, whether they are published or not. The documents may come from teaching and research institutions in France or abroad, or from public or private research centers.
L'archive ouverte pluridisciplinaire HAL, est destinée au dépôt et à la diffusion de documents scientifiques de niveau recherche, publiés ou non, émanant des établissements d'enseignement et de recherche français ou étrangers, des laboratoires publics ou privés. 


\section{Les grandeurs de référence utilisables dans l'étude de l'alimentation en eau des cultures}

Nader KATERJI \& Marc HALLAIRE

I.N.R.A., Station de Bioclimatologie, Route de St-Cyr, F 78000 Versailles

Les recherches concernant l'alimentation en eau des cultures et ses conséquences sur la croissance ou le rendement requièrent la détermination de 2 types de grandeurs :

- Un $1^{\text {er }}$ type de grandeurs $(\mathrm{X})$, caractérisant à tout instant l'état hydrique du végétal.

- Un $2^{\mathrm{e}}$ type $(\mathrm{Y})$ relatif au milieu ou au végétal lui-même et définissant les conditions d'alimentation hydrique.

En ce qui concerne le $1^{\text {er }}$ type de grandeurs $(\mathrm{X})$, on a souligné l'intérêt que présente le potentiel foliaire $\Psi_{\mathrm{F}}$. Dès lors, les grandeurs de type $\mathrm{Y}$ à connaître sont celles qui déterminent $\Psi_{\mathrm{F}}$. Or, une modélisation des transferts hydriques dans l'ensemble sol-plante (modélisation proposée antérieurement et rappelée ici) permet précisément de calculer la cinétique journalière de $\Psi_{\mathrm{F}}$. Les grandeurs $\mathrm{Y}$ ne seront autres que les facteurs pédoclimatiques et les paramètres du modèle pris en compte dans le calcul de $\Psi_{\mathrm{F}}$. Ce sont :

- les transpirations réelles horaires $\mathrm{T}$,

- le potentiel foliaire de base $\Psi_{\mathrm{Fb}}$ (potentiel d'équilibre avec le sol, mesurable en fin de nuit),

- les 4 paramètres introduits dans le modèle (fig. 3) et qui caractérisent essentiellement le végétal, à savoir :

- la résistance $\mathrm{R}$ du circuit principal sol-feuille (qui dépend de l'extension racinaire et de l'humidité du sol),

- une résistance interne $r$,

- 2 caractéristiques du réservoir-plante $\left(\Psi_{\mathrm{pc}}\right.$ et $\mathrm{V} / \rho$ cf. fig. 3$)$ qui découlent de la relation (teneur en eau relative - potentiel du réservoir) et conditionnent la participation de cette réserve à la transpiration journalière.

On a pu apprécier et illustrer les poids relatifs des 2 facteurs pédoclimatiques ( $T$ et $\Psi_{\mathrm{Fb}}$ ) et des 4 paramètres du modèle $\left(R, r, \Psi_{p c}\right.$ et $\left.V / \rho\right)$ sur la cinétique de $\Psi_{F}$ (fig. 6 ) et, par voie de conséquence, sur les durées de fonctionnement optimal de la feuille.

Mots clés additionnels : Potentiel hydrique foliaire, modélisation

Research concerned with water needs in crops and its consequences on growth and yield requires two types of measure :

- a first type of measure (X) characterizing plant water content at each instant,

- a second type of measure $(\mathrm{Y})$ relative to the environment or to the plant itself and defining the conditions of water supply.

As concerns the first type of measure (X), we have stressed the interest which leaf potential $\Psi_{\mathrm{F}}$ presents. The Y-type measures we must then know are those which determine $\Psi_{\mathrm{F}}$. In fact, a model of water transfer in the soilplant system (proposed earlier and recalled here) can allow daily changes in $\Psi_{\mathrm{F}}$ to be calculated precisely. The $Y$ measures, then, can be simply the pedoclimatic factors and the parameters of the model taken into consideration in calculating $\Psi_{\mathrm{F}}$. They are :

- hourly actual transpiration $\mathrm{T}$,

- predawn leaf potential $\Psi_{\mathrm{Fb}}$ (potential of equilibrium with the soil, measured at the end of the night),

- the four parameters introduced into the model (fig. 3) and which essentially characterize the plant. These four parameters are :

- the resistance $\mathrm{R}$ of the principal soil-leaf circuit which depends upon root extension and soil humidity,

- an internal resistance $r$,

- two characteristics of plant storage capacity $\left(\Psi_{\mathrm{pc}}\right.$ et $\mathrm{V} / \mathrm{\rho}$ fig. 3) which ensue from the (relative water content - storage potential) relationship and condition the participation of this reserve in daily transpiration.

We were able to assess and illustrate the relative weights of the two pedoclimatic factors $\left(T\right.$ and $\left.\Psi_{F b}\right)$ and the four parameters of the model $\left(R, r, \Psi_{p c}\right.$ and $\left.V / p\right)$ on the change in $\Psi_{F}$ (fig. 6) and, consequently, on the duration of optimal leaf function.

Additional key words : Leaf water potential, model 


\section{INTRODUCTION}

Le rôle essentiel de l'eau comme facteur de la production végétale justifie l'importance des recherches consacrées à ce thème, comme le montre par exemple l'historique réalisé par BOYER (1976).

Dans l'étude des relations de cause à effet que l'on tente de mettre en évidence, on est amené à se référer à 2 types de grandeurs :

- Les grandeurs du $1^{\text {er }}$ type, que l'on désignera ici par $\mathrm{X}$, visent à exprimer l'état hydrique de la plante (potentiel hydrique, déficit de saturation d'un organe, résistance stomatique...) ou permettent d'apprécier, sur les laps de temps plus ou moins longs, dans quelle mesure les besoins en eau du végétal ont été satisfaits (rapport des évapotranspirations réelle et potentielle).

- Les grandeurs du $2^{\mathrm{e}}$ type, désignées ici par $\mathrm{Y}$, sont celles qui, caractérisant le milieu ou encore certaines propriétés physiques ou anatomiques du végétal, conditionnent l'équilibre hydrique de la plante (demande climatique (ETP), stock d'eau disponible, potentiel hydrique du sol, développement racinaire, conductivité des milieux traversés...).

Les études consistent alors le plus souvent à rechercher, soit l'influence de la grandeur $X$ sur l'assimilation, la croissance, le développement, la production, éventuellement certains facteurs de qualité, soit l'influence des différentes grandeurs $Y$ sur la grandeur $\mathrm{X}$ retenue.

Dans le présent article, nous nous proposons :

- de passer en revue les différentes grandeurs ( $\mathrm{X}$ et $\mathrm{Y}$ ) habituellement considérées,

- de souligner l'intérêt de retenir le potentiel foliaire $\Psi_{\mathrm{F}}$ comme critère de type X,

- d'indiquer, sur la base d'une modélisation proposée antérieurement (KATERJI et al., $1983 a$ et $b$, 1984), l'ensemble des paramètres mesurables qui, déterminant $\Psi_{\mathrm{F}}$ à tout instant, méritent d'être retenus comme critères de type $(\mathrm{Y})$.

\section{LES GRANDEURS DE RÉFÉRENCE COURAMMENT UTILISÉES}

\section{A. Les grandeurs (X) exprimant l'état hydrique du végétal}

Les grandeurs en question peuvent être des expressions directes ( $\$ 1$ et 2 ) ou indirectes ( $\$ 3$ et 4 ) de l'état hydrique considéré.

\section{Grandeurs liées à la teneur en eau du végétal}

La teneur en eau (masse d'eau rapportée à l'unité de masse de matière sèche) constitue une référence incommode, sa gamme de variation étant éminemment variable d'un organe à l'autre ; aussi préfère-ton retenir soit la teneur en eau relative RWC (relative water content $)=\mathrm{v} / \mathrm{V}(\mathrm{v}=$ volume d'eau présent ; $\mathrm{V}=$ valeur maximale de $\mathrm{v}$ c'est-à-dire à pleine turgescence), soit encore le déficit de saturation WSD (water saturation deficit), valeur complémentaire de la précédente $(\mathrm{WSD}=1-\mathrm{v} / \mathrm{V})$.
On rappellera les difficultés d'ordre technique concernant notamment la détermination exacte de $\mathrm{V}$. On soulignera par ailleurs que la détermination de ces grandeurs implique des mesures manuelles et, par conséquent, l'intervention de l'observateur au moment même où l'on veut connaître cet état hydrique.

Les techniques de mesures relatives (cf. revue bibliographique in BURIOL, 1981) présentent l'inconvénient d'être soit inapplicables au champ, soit très imprécises, soit enfin pour la plupart d'être manuelles et assez laborieuses.

\section{Le potentiel hydrique d'un organe végétal}

Cette grandeur qui définit l'état énergétique de l'eau est le meilleur indicateur de l'état hydrique de la plante (BERGER, 1970 ; HSIAO, 1976).

Ce paramètre s'est largement imposé par rapport aux autres critères précédemment rappelés dans les recherches touchant à l'influence de l'état hydrique du végétal sur la transpiration, la photosynthèse, la croissance, la respiration, le transfert des assimilats (BOYER, 1976 ; Hsiao et al., 1976 ; LudLOW, 1976 ; RoY, 1980). Trois raisons justifient cet intérêt :

Le $\Psi_{F}$ traduit physiquement l'état énergétique de l'eau dans la plante tandis que les autres paramètres n'en donnent qu'une indication relative (BERGER, 1970).

- Certaines fonctions de la plante comme la résistance stomatique, la différenciation et l'élongation cellulaire semblent liées directement à $\Psi_{\mathrm{F}}$ (LuDLOW, 1976 ; ROY, 1980) ou plus précisément à l'une de ses composantes, le potentiel de turgescence (BEGG \& TURNER, 1976).

- Réalisable à l'aide d'une chambre à pression, la mesure de $\Psi_{F}$ est beaucoup plus rapide que celle de RWC ou de WSD. De plus, les études statistiques réalisées notamment sur luzerne (KATERJI, 1982) et sur pomme de terre (KATERJI, résultats non publiés), concernant la variation systématique et la variabilité aléatoire des données de $\Psi_{\mathrm{F}}$ obtenues à l'intérieur de la végétation, montrent que l'erreur relative sur un échantillon de 10 mesures de $\Psi_{F}$ n'est que de 10 p. 100 environ.

Son inconvénient est que sa mesure n'est toujours pas automatisable en milieu naturel.

\section{La résistance stomatique $r_{s}$}

En l'absence de régulation photique liée à un éclairement insuffisant (KANEMASU \& TANNER, 1969; Mc Pherson \& Slatyer, 1973), l'accroissement de $r_{s}$ traduit généralement un dessèchement relatif des feuilles et conduit à une réduction de la transpiration végétale et de l'assimilation. Ainsi est-elle apparue longtemps comme un critère particulièrement valable pour exprimer les conditions momentanées d'alimentation hydrique. Cependant, et bien que dans beaucoup de travaux on constate une chute de l'assimilation parallèle à l'augmentation de la résistance stomatique (BRIX, 1962 ; WILlis \& BALASUBRAMANIAN, 1968 ; MOLDAU, 1972 ; SLATYER, 1973), certains auteurs (SHIMSHI, 1963 ; TROUGHTON, 1969 ; BOYER, 1971 ; 
HANSEN, 1971 ; LUDLOW \& NG, 1976) suggèrent que la chute de la photosynthèse ne proviendrait pas uniquement de l'accroissement de la résistance stomatique, mais serait attribuable en partie à un accroissement de la résistance non stomatique au transfert de $\mathrm{CO}_{2}$ (résistance au transfert de $\mathrm{CO}_{2}$ dans le mésophylle et résistance à la carboxylation). Des preuves directes de cette hypothèse ont été apportées grâce à l'utilisation de techniques permettant de déterminer cette résistance interne (GRAZIANI \& LIVNE, 1971 ; MEDERSKI et al., 1975).

Ce paramètre demeurerait toutefois une donnée de référence intéressante s'il était facilement et rapidement mesurable, ce qui est loin d'être le cas ; $r_{s}$ présente en effet, d'une part, des variations spatiales importantes selon l'âge de la feuille (TURNER, 1969), 1'éclairement à l'intérieur même du couvert (KATERJI et al., 1983c), ainsi qu'à l'échelle même de la feuille et, d'autre part, des variations systématiques dans le temps (BARRS, 1971 ; COWAN, 1972). La détermination précise de la résistance à l'échelle du couvert requiert ainsi la réalisation d'un grand nombre de mesures, lesquelles de surcroît ne sont pas automatisables.

La détermination de $r_{s}$ peut se déduire également des mesures simultanées de l'évapotranspiration réelle du couvert, du rayonnement net, du déficit hydrique et de la température de l'air ainsi que de la résistance aérodynamique au-dessus du couvert (MONTEITH, 1965 ; PERRIER, 1975). La grandeur théorique obtenue offre le double avantage d'être caractéristique de l'ensemble du couvert et d'être déduite d'un ensemble de grandeurs enregistrables. Par contre la relation entre cette grandeur calculée et la résistance stomatique moyenne du couvert n'a pas encore été suffisamment explicitée (CRUZIAT et al., 1979; KATERJI, 1982).

\section{Le rapport des évapotranspirations réelle et poten- tielle}

De nombreuses études (BOUCHET \& ROBELIN, 1969 ; PUECH et al., 1976) expriment la croissance ou le rendement agricole en fonction des rapports ETR/ETP ou ETR/ETR ${ }_{M}$, expressions dans lesquelles :

- ETR représente l'évapotranspiration réelle (obtenue par pesée de cases ou de pots de végétation, par la méthode aérodynamique, celle du bilan énergétique ou celle du bilan hydrique).

- ETP représente l'évapotranspiration potentielle calculée (formule de PENMAN par exemple).

- $\mathrm{ETR}_{\mathrm{M}}$ représente l'évapotranspiration maximale du couvert considéré, c'est-à-dire l'ETR en conditions d'alimentation hydrique optimales.

L'intérêt de cette grandeur de référence se justifie à beaucoup de points de vue : les grandeurs à mesurer sont enregistrables. Selon l'objectif recherché, le rapport considéré s'appliquera à des laps de temps pouvant aller de l'heure à la saison complète de végétation, de même qu'au niveau spatial, il peut aussi bien caractériser une feuille, une plante, un couvert végétal voire même le bassin versant envisagé dans les études d'hydrologie.
A l'échelle instantanée, le rapport ETR/ETR $\mathrm{ET}_{\mathrm{M}}$ a sans doute à peu près la même signification que la résistance stomatique moyenne du couvert et, à ce titre, peut effectivement faire l'objet des mêmes remarques : signification essentiellement en terme de transpiration mais pas forcément reliable à d'autres aspects de la physiologie ; de plus, il est un reflet très imparfait de la résistance stomatique quand l'évaporation directe du sol constitue une fraction non négligeable de l'ETR.

En définitive, parmi les grandeurs de type $X$, le potentiel hydrique $\Psi_{\mathrm{F}}$ apparaît comme un critère particulièrement intéressant pour analyser le rôle de l'état hydrique sur certaines fonctions de la plante comme la résistance stomatique. De plus, lorsqu'il s'agit d'analyser la chute de photosynthèse en fonction du stress hydrique, il est souhaitable que cette analyse se fasse également en fonction de $\Psi_{\mathrm{F}}$ pour tenir compte de l'existence éventuelle d'une inhibition de la photosynthèse autre que stomatique. Notons enfin que, dans les modèles de fonctionnement du couvert (SAUGER, 1974) qui lient l'assimilation nette exclusivement à $r_{s}$, le potentiel hydrique reste une donnée essentielle puisque $r_{s}$ est déterminée en partie par $\Psi_{F}$.

\section{B. Les grandeurs (Y) exprimant les conditions d'ali- mentation hydrique}

\section{Le potentiel moyen du sol $\Psi_{s}$ et le potentiel foliaire de base à l'état d'équilibre $\Psi_{F b}$}

- Lorsque les racines explorent un milieu homogène dont le potentiel présente une valeur unique $\Psi_{s}$, on peut vérifier (MAERTENS, 1981) que le potentiel foliaire à l'équilibre correspond à $\Psi_{\mathrm{s}}$ (à l'erreur de mesure près soit environ 1 bar).

- Dans les conditions du champ où les $n$ couches de sol explorées par les racines présentent des potentiels différents $\left(\Psi_{\mathrm{s}}\right)_{1},\left(\Psi_{\mathrm{s}}\right)_{2} \ldots\left(\Psi_{\mathrm{s}}\right)_{\mathrm{n}}$, plusieurs auteurs ont proposé d'exprimer le potentiel moyen du sol par la relation :

$$
\bar{\Psi}_{s}=\sum_{i=1}^{n}\left(\Psi_{s}\right)_{i} X_{i} / \sum_{i=1}^{n} X_{i}
$$

le terme $\mathrm{X}_{\mathrm{i}}$ désignant pour la tranche (i) soit la masse (VAN BAVEL \& AHMED, 1976) soit la longueur des racines (MAERTENS, 1981).

Il a été suggéré par ailleurs (KLEPPER, 1968 ; WARING, 1969 ; JORDAN \& RITCHIE, 1971 ; SUCOFF, 1972) ou vérifié (MAERTENS, 1981 ; STEINHARDT et al., 1981 : KATERJI et al., 1983a) que le potentiel foliaire à l'équilibre $\Psi_{\mathrm{Fb}}$ était très voisin de la valeur de $\Psi_{\mathrm{s}}$ ainsi définie :

$$
\Psi_{\mathrm{Fb}}=\bar{\Psi}_{\mathrm{s}} \pm 1 \text { bar } .
$$

Cette valeur représente un critère de type $Y$ d'un intérêt évidẹnt.

\section{Les conditions d'équilibre entre l'offre et la demande en eau}

- Lorsque les racines explorent un milieu homogène, il a pu être montré et vérifié qu'en première 
approximation (HALlAIRE, 1964) le flux liquide maximal du sol aux racines est donné par :

$$
\mathrm{q}_{\max }=\alpha\left(\mathrm{W}-\mathrm{W}_{\mathrm{f}}\right)
$$

W désignant l'humidité du sol, $\mathrm{W}_{\mathrm{f}}$ le point de flétrissement permanent et $\alpha$ un coefficient de proportionnalité, fonction croissante de la diffusivité $\mathrm{D}$ de l'eau dans le sol et de la densité racinaire $\mathrm{d}_{\mathrm{r}} ; \mathrm{q}_{\max }$ qui

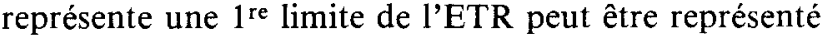
en fonction de W (fig. 1a) par des droites telles que $\mathrm{D}_{1}$ et $\mathrm{D}_{2}$.

Sur le même graphique ( $1 a)$, on a figuré cette autre valeur limite de l'ETR que représente l'évapotranspiration potentielle instantanée (droites $\mathrm{ETP}_{1}$ et $\mathrm{ETP}_{2}$ ). A tout moment, la limite supérieure de l'ETR correspondra à la plus petite des 2 valeurs limites $\mathrm{q}_{\max }$ et ETP.

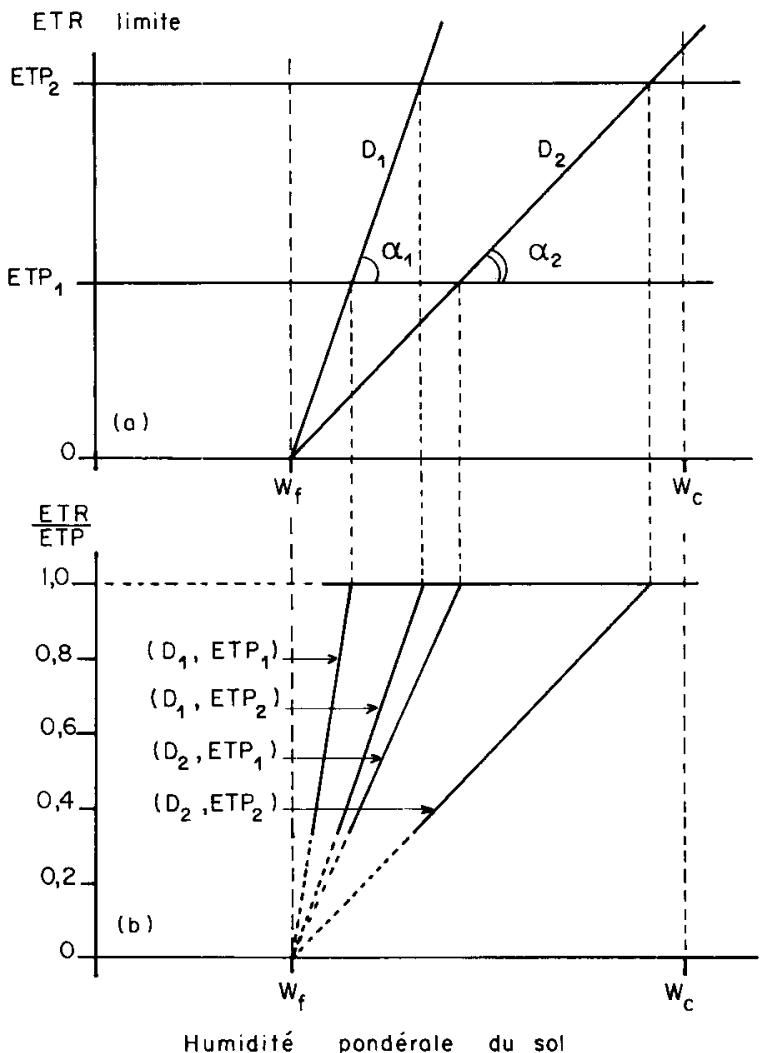

Figure 1

a) Limites supérieures de l'ETR (droites ETP,$E T P_{2}$ : évapotranspiration potentielle; droites $D_{l}, D_{2}:$ flux d'eau accessible aux racines) en fonction de l'humidité du sol $W$.

b) Variation du rapport ETR/ETP en fonction de l'humidité du sol $W$.

a) Upper limits of ETR (lines ETP,$E T P_{2}$ : potential evapotranspiration; lines $D_{l}, D_{2}$ : water flow accessible to roots) in relation to soil humidity.

b) Variation in the ETR/ETP ratio in relation to soil humidity.

Si l'on recherche alors la relation entre cette grandeur de type $X$ que représente le rapport ETR/ETP, et l'humidité $W$, on aboutit à des courbes aussi différentes que celles représentées figure $1 b$; l'humidité critique au-dessous de laquelle il y a déséquilibre entre l'offre et la demande est d'autant plus faible que l'ETP est plus faible et que $D$ et $d_{r}$ sont plus grands. Les facteurs (Y) à retenir sont donc l'ETP, l'humidité $W$, la diffusivité $D$ et la densité racinaire $\dot{d}_{r}$.
- Dans les conditions de plein champ où l'humidité du sol n'est plus homogène, il est évident que les facteurs $Y$ conduisant à la satisfaction des besoins en eau de la plante ou au contraire à un certain déséquilibre, dépendront non seulement du potentiel de base $\left(\Psi_{\mathrm{Fb}}=\bar{\Psi}_{\mathrm{s}}\right)$ précédemment défini mais également de la résistance aux transferts de l'eau du sol à la feuille et éventuellement de la mobilisation des réserves contenues dans le végétal.

Un modèle de transfert de l'eau dans le végétal, proposé antérieurement, permet précisément de définir et d'évaluer les différentes grandeurs (de type $Y$ ), dont dépend cette grandeur (de type $X$ ) qu'est le potentiel foliaire $\Psi_{\mathrm{F}}$.

\section{CINÉTIQUE JOURNALIÈRE ET MODÉLISATION DU POTENTIEL HYDRIQUE FOLIAIRE $\Psi_{\mathrm{F}}$}

\section{A. Cinétique observée de $\Psi_{F}$ et relation avec la trans- piration $T$}

Les nombreux résultats expérimentaux indiqués dans la littérature (BERGER, 1971 ; REISCOSKY et al.,
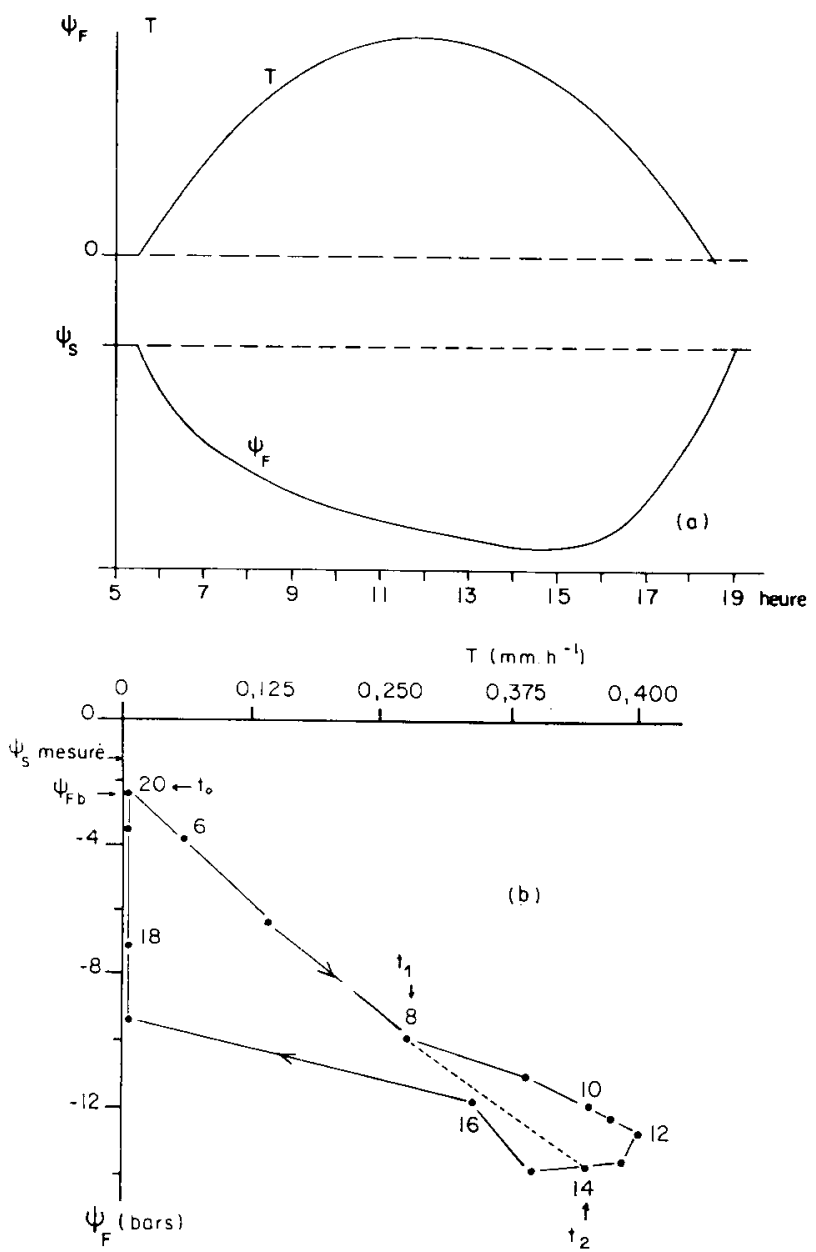

Figure 2

a) Variations journalières de la transpiration $T$ et du potentiel foliaire $\psi_{F}$.

b) Relation trouvée sur une culture de luzerne entre le potentiel foliaire $\psi_{F}$ et la transpiration $T$.

a) Daily variation in transpiration $T$ and leaf potential $\psi_{F}$

b) Relationship between leaf water potential and transpiration rate in a lucerne crop. 
1982) ou obtenus par nous-mêmes (KATERJI et al., $1983 a$ ) peuvent se résumer comme suit :

Portant sur un graphique les valeurs mesurées de $\Psi_{\mathrm{F}}$ aux différentes heures du jour, on note (fig. 2a) que :

- $\Psi_{F}$ est généralement constant durant les dernières heures de la nuit. Cette valeur n'est autre que le potentiel de base $\Psi_{\mathrm{Fb}}$ dont il a été question plus haut ; on a vu qu'il correspond au potentiel moyen du sol dans la zone racinaire ; on la désignera donc par $\Psi_{s}$,

- l'amplitude de $\Psi_{\mathrm{F}}$ peut dépasser par belle journée une dizaine de bars,

- contrairement à la courbe de transpiration qui, par journée claire du moins, est symétrique autour de $12 \mathrm{~h}$, la courbe $\Psi_{\mathrm{F}}$ est dissymétrique et présente un maximum vers $14-15 \mathrm{~h}$.

Ce dernier résultat, signifiant que $\Psi_{\mathrm{S}}-\Psi_{\mathrm{F}}$ et $\mathrm{T}$ ne restent pas toujours proportionnels, suggère de représenter graphiquement $\Psi_{F}$ en fonction de T. On constate alors (fig. 2b) que :

- par belle journée : $\Psi_{\mathrm{F}}$ varie en fonction de $\mathrm{T}$ de façon linéaire du lever du soleil (temps $\mathrm{t}_{0}$ ) jusqu'à un temps $t_{1}\left(8 \mathrm{~h}\right.$ dans le cas de la fig. $2 \mathrm{~b}$ ); $\Psi_{5}-\Psi_{\mathrm{F}}$ apparaît alors proportionnel à $T$. De $t_{1}$ à $t_{2}\left(t_{2}=14 \mathrm{~h}\right.$, fig. 2b), les points se situent au-dessus de la droite trouvée précédemment. Au-delà de $t_{2}$, ils se situent au-dessous ;

- par journée couverte comme par début de belle journée $\left(\mathrm{t}_{0}-\mathrm{t}_{1}\right)$, la transpiration restant faible, la relation $\Psi_{F}(\mathrm{~T})$ demeure linéaire.

\section{B. Modélisation du transfert d'eau liquide du sol à la feuille}

La loi classique selon laquelle le flux liquide est proportionnel à la différence de potentiel entre les 2 bornes du circuit considéré (VAN DEN HONERT, 1948) apparaît ainsi en défaut durant la majeure partie des journées ensoleillées. Plusieurs hypothèses ont été proposées par ailleurs et nous en avons fait la critique dans un article précédent (KATERJI et al., 1983a). L'interprétation que nous avons proposée repose sur le modèle de transfert représenté figure $3 a$ qui revient à admettre la présence, en marge du circuit principal (sol-feuille), d'un réservoir susceptible alternativement de céder et de récupérer de l'eau.

A tout moment, les potentiels à considérer sont $\Psi_{s}$ pour le sol, $\Psi_{\mathrm{F}}$ pour la feuille et $\Psi_{\mathrm{p}}$ pour le réservoirplante. Les flux sont la transpiration (T) dans la partie supérieure du circuit principal, l'absorption racinaire (a) à sa partie inférieure et la contribution du réservoir-plante (q), ces 3 flux étant reliés entre eux par l'équation :

$$
\mathrm{T}=\mathrm{a}+\mathrm{q} \text {. }
$$

Les paramètres $d u$ modèle sont tout d'abord les résistances $R$ et $r$ qui permettent d'exprimer la loi d'Ohm pour les 2 parcours correspondants (fig. 3a)

$$
\Psi_{\mathrm{s}}-\Psi_{\mathrm{r}}=\mathrm{R} \cdot \mathrm{a}
$$

( $R$ intégrant essentiellement les résistances au transfert de l'eau dans les manchons du sol entourant les racines et la résistance à la pénétration radiale dans les racines)

et

$$
\Psi_{p}-\Psi_{F}=\text { r.q. }
$$

Ce sont, d'autre part, les caractéristiques hydrodynamiques du réservoir : si l'on imagine (fig. 3c) la courbe exprimant la variation de $\Psi_{\mathrm{p}}$ en fonction de l'humidité relative du réservoir $\mathrm{v} / \mathrm{V}$ et si l'on désigne par $\rho$ la pente, on peut établir la relation suivante entre la variation de $\Psi_{\mathrm{p}}$ et le débit $\mathrm{q}$ :

$$
q=-\frac{d v}{d t}=-\frac{V}{\rho} \frac{d \Psi_{p}}{d t} .
$$

Les 2 paramètres $V$ et $\rho$ caractéristiques du réservoir n'interviennent donc que par leur rapport $V / \rho$.

En ce qui concerne la pente $\rho$, nous avons vérifié (KATERJi et al., 1983a) que, pour la luzerne :

- $\rho$ est très grand (d'où $\mathrm{V} / \rho$ \# 0 ) dans la gamme des $\Psi_{\mathrm{p}}$ supérieurs à une valeur critique $\Psi_{\mathrm{pc}}$. Celle-ci est apparue stable dans le cas de la luzerne $\left(\Psi_{\mathrm{pc}}=-8,5\right.$ bars $)$ tandis que, pour la pomme de terre, elle présente des

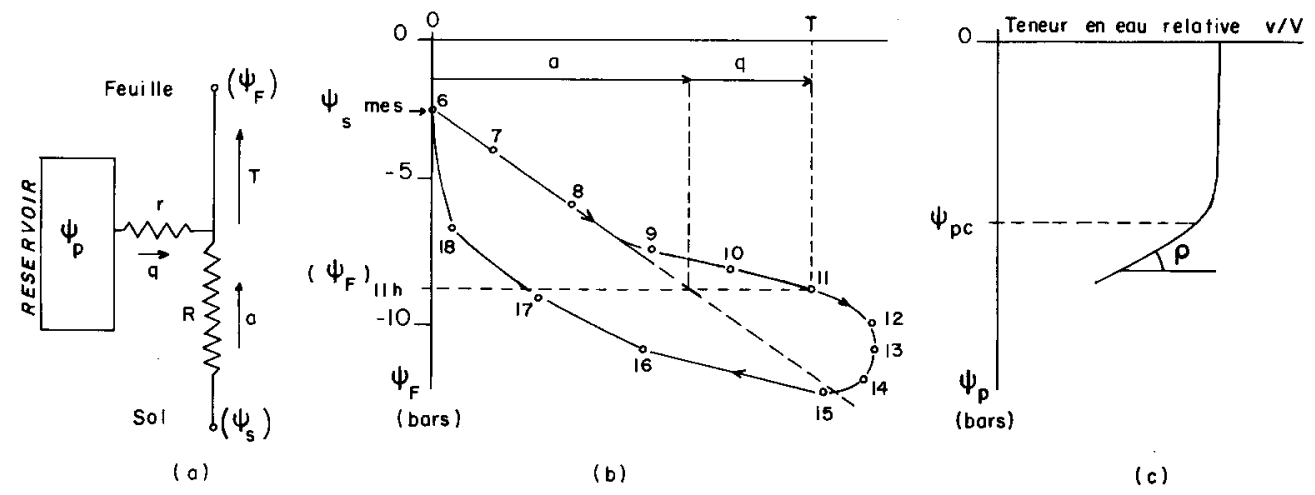

Figure 3

Modèle de circulation de l'eau dans la plante et interprétation de la relation trouvée entre $\psi_{F}$ et $T$.

a) Schéma du modèle de circulation.

b) Relation entre le potentiel hydrique foliaire et la transpiration $T$; mise en évidence du flux interne $q$ à $11 \mathrm{~h}$.

c) Relation entre le potentiel du réservoir $\psi_{p}$ et sa teneur en eau relative $v / V$; mise en évidence de la gamme de potentiel $\left(0-\psi_{p d}\right)$ pour laquelle les variations de $\psi_{p}$ correspondent à un flux $q$ nul.
Model of water circulation within plants and interpretation of the relationship found between $\psi_{F}$ and $T$.

a) Diagram of the circulation model.

b) Relationship between leaf potential $\left(\psi_{F}\right)$ and transpiration $(T)$; the internal flow $(q)$ at a given hour (11.00) is shown.

c) Relationship between potential $\left(\psi_{p}\right)$ and relative humidity $(\nu / V)$ of the reservoir; the range of potentials $\left.10-\psi_{p d}\right)$ for which the variations in $\Psi_{p}$ are accompanied by a zero $q$ flow is shown. 
variations systématiques au cours de la croissance à l'intérieur des limites $-6 \pm 1,3$ bars ;

- $\rho$ est pratiquement constant (d'où $V / \rho \# C^{\text {te }}$ ) dans la gamme des $\Psi_{n}$ inférieure à la valeur critique $\Psi_{\mathrm{pc}}$.

Ce résultat peut être rapproché de données expérimentales sur la relation $\Psi_{F}=f(v / V)$ qui présente généralement les mêmes caractéristiques (CRUIZIAT, 1978 ; PICOT, 1983).

En définitive, les 4 paramètres caractérisant le modèle sont $\mathrm{R}, \mathrm{r}, \Psi_{\mathrm{pc}}$ et la valeur de $\mathrm{V} / \rho$ correspondant à $\Psi_{\mathrm{p}}<\Psi_{\mathrm{pc}}$.

\section{Interprétation des résultats sur la base du modèle}

Selon le modèle, la partie linéaire de la courbe observée sur le graphe donnant $\Psi_{\mathrm{s}}-\Psi_{\mathrm{F}}$ en fonction de la transpiration $\mathrm{T}$ (fig. 2b) ainsi que son prolongement vers les $\Psi_{F}$ bas ne serait autre que l'expression graphique de l'équation (3), le coefficient angulaire de cette droite correspondant par conséquent à la résistance $\mathrm{R}$. Dès lors :

- Au cours de la phase $\mathrm{t}_{0}-\mathrm{t}_{1}$, les points portés figure $2 \mathrm{~b}$ se placent sur la droite précitée parce que, $\Psi_{\mathrm{F}}$ et $\Psi_{\mathrm{p}}$ étant supérieurs à la valeur critique $\Psi_{\mathrm{pc}}$,

- $\mathrm{V} / \rho$ est assimilable à 0 (cf. fig. $3 \mathrm{c}$ ),

- q est nul (cf. équation (5)),

- et la transpiration (T) portée en abscisse se confond alors avec l'absorption racinaire (a) (cf. équation (2)).

- De $\mathrm{t}_{1}$ au coucher du soleil, les points observés sur le graphe de la figure $2 b$ s'écartent de la droite en question parce que, $\Psi_{\mathrm{p}}$ étant inférieur à $\Psi_{\mathrm{pc}}$,

- $\mathrm{V} / \rho$ est différent de 0 ,

- q n'est plus nul,

- et la transpiration (T) ne correspond plus au flux (a).

Dans ces conditions, l'abscisse de la droite extrapolée et relative à un $\Psi_{\mathrm{F}}$ donné $\left(\Psi_{\mathrm{F}}\right.$ observé à $11 \mathrm{~h}$ dans le cas de la figure $3 b$ ) correspond toujours à l'absorption racinaire (a) et la différence d'abscisses entre la courbe et la droite représente la contribution du réservoir-plante (q). De $t_{1}$ à $t_{2},(9 \mathrm{~h}$ à $15 \mathrm{~h}$, fig. $3 \mathrm{~b})$, q est positif, ce qui signifie que le réservoir cède son eau. Après $t_{2}$ (soit $15 \mathrm{~h}$, fig. 3b), q étant négatif, le réservoir se remplit à nouveau.

\section{Détermination des 4 paramètres du modèle (phase de calibrage)}

Ainsi qu'il a été explicité par ailleurs (KATERJI et al., 1983a), supposant connues les variables d'entrée (VE) déterminées au cours d'une journée claire :

- VE : $\Psi_{\mathrm{s}}-\Psi_{\mathrm{F}}$ et $\mathrm{T}$ horaires du lever du soleil et jusqu'à équilibre $\left(\Psi_{\mathrm{F}}=\Psi_{\mathrm{s}}\right.$, on peut déduire les variables de sortie (VS) représentées par les 4 paramètres du modèle ;

- VS : R, r, V/ $\rho$ et $\Psi_{\mathrm{pc}}$ dans la mesure toutefois où ces 4 paramètres ( $R, r$ en particulier) ne varient pas d'une façon appréciable au cours de la journée.

Rappelons qu'au plan technique l'acquisition des variables d'entrée requiert :

- un équipement de mesure de l'évapotranspiration réelle du type BEARN (PERRIER et al., 1976), ou
SAMER (ITIER, 1981) ou d'un évapotranspiromètre pesable,

- une chambre à pression pour obtenir $\Psi_{\mathrm{Fb}}$ (égal à $\Psi_{\mathrm{s}}$ ) et les valeurs horaires de $\Psi_{\mathrm{F}}$.

Notons également que les transpirations horaires $T$ sont assimilables, à un léger correctif près, aux ETR mesurées dans le cas des cultures bien couvrantes; par contre, l'obtention exacte de la transpiration $T$ peut poser un problème difficile dans le cas de cultures peu couvrantes comme la vigne ou les jeunes vergers, la transpiration pouvant alors être très inférieure à l'ETR mesurée.

\section{E. Détermination par calcul des cinétiques journaliè- res des potentiels $\Psi_{F}$ et $\Psi_{p}$ et des débits (phase d'utilisation)}

Inversement, ainsi qu'il a été explicité par ailleurs (KATERJI et al., 1983a), ayant déterminé les 4 paramètres du modèle à partir d'une journée et disposant des variables d'entrée (VE) suivantes :

- VE : $\Psi_{\mathrm{s}}$ et $\mathrm{T}$ horaire et $\mathrm{R}, \mathrm{r}, \mathrm{V} / \rho$ et $\Psi_{\mathrm{pc}}$, on peut déduire pour toute autre journée les variables de sortie (VS) représentées par les 4 fonctions suivantes :

- VS : $\Psi_{\mathrm{p}}(\mathrm{t})$, a $(\mathrm{t})$, q $(\mathrm{t})$, et en particulier $\Psi_{\mathrm{F}}(\mathrm{t})$ objet de la présente étude.

En effet, la solution du système d'équations (2), (3), (4) et (5) conduit à l'équation différentielle

$$
\Psi_{s}-\Psi_{p}=R T+\frac{V}{\rho}(R+r) \frac{d \Psi_{p}}{d t}
$$

équation qui, dans le cas particulier où $\frac{V}{\rho}=0$, se ramène à

$$
\Psi_{\mathrm{s}}-\Psi_{\mathrm{p}}=\mathrm{RT} \text {. }
$$

On peut ainsi calculer $\Psi_{p}$ (d'heure en heure par ex.),

- dans un $1^{\text {er }}$ temps sur la base de l'équation $6^{\prime}$ (lorsque $\Psi_{\mathrm{p}}>\Psi_{\mathrm{pc}}$ d'où $\mathrm{V} / \rho=0$ ),

- dans un $2^{\mathrm{e}}$ temps (lorsque $\Psi_{\mathrm{p}}<\Psi_{\mathrm{pc}}$ et que $\frac{\mathrm{V}}{\rho}$ a la valeur constante connue), en intégrant de proche en proche l'équation (6),

- enfin, dans un $3^{\text {e }}$ temps sur la base de $6^{\prime}$ (quand $\Psi_{\mathrm{p}}$ est redevenu supérieur à $\Psi_{\mathrm{pc}}$ ).

Connaissant l'évolution journalière de $\Psi_{p}$, on en déduit immédiatement $\mathrm{d} \Psi_{\mathrm{p}} / \mathrm{dt}$, d'où les valeurs horaires de q (équation 5), de a (équation 2) et enfin de $\Psi_{\mathrm{F}}$ (équation 3).

\section{F. Vérifications expérimentales du modèle}

- La résistance $\mathrm{R}$ d'un couvert de luzerne (évaluée chaque jour par la pente du segment de droite du graphe donnant $\Psi_{F}$ en fonction de $T$ ) (cf. fig. 2b) est apparue constante à 10 p. 100 près au cours de 26 journées pendant lesquelles l'humidité du sol demeurait elle-même assez stable (KATERJI et al., $1983 b)$. En outre, les variations attendues de $\mathrm{R}$ en fonction de la sécheresse édaphique semblent être confirmées par une $1^{\text {re }}$ série de résultats obtenus sur un culture de pomme de terre (résultats non publiés).

- Les 4 paramètres caractéristiques du couvert de 
luzerne (déterminés à partir des valeurs horaires de $\Psi_{F}$ et $T$, mesurées par une belle journée comme indiqué au $\S \mathrm{D})$ ont permis d'établir par calcul la cinétique de $\Psi_{\mathrm{F}}$ au cours de 3 autres belles journées.

La correspondance s'avère bonne entre les valeurs calculées et mesurées (fig. 4).

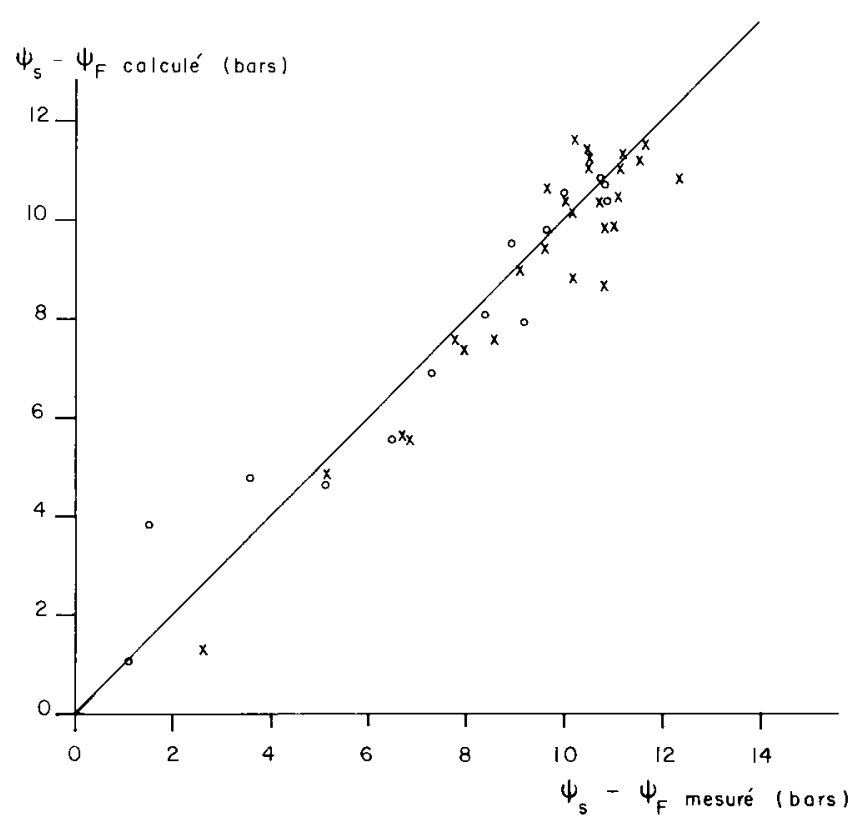

Figure 4

Comparaison des valeurs de $\psi_{F}-\psi_{s}$ mesurées aux valeurs calculées par le modèle. Les points correspondent à la journée qui a permis d'établir les paramètres du modèle, les croix à 3 autres journées.

Comparison between the measured values of $\psi_{s}-\psi_{F}$ and those calculated by the model. The points correspond to the day used to establish the parameters of the model and the crosses to three other days.

- Le modèle permet d'évaluer les débits q horaires et, en additionnant les valeurs positives de ces derniers, d'évaluer la quantité d'eau totale fournie par la plante au cours d'une journée. Dans le cas de la luzerne (KATERJI et al., 1983b), cette perte totale varie de 0 à 15 p. 100 environ de l'évapotranspiration journalière lorsque cette dernière varie de 2 à $5 \mathrm{~mm}$. Ce résultat semble tout à fait cohérent. D'autre part, on a constaté que les pertes totales ( $\Sigma \mathrm{q}$ positif) données par le modèle (et qui concernent donc l'ensemble des parties souterraines et aériennes) sont environ 3 fois plus élevées que les pertes de la partie aérienne mesurées par les hyperfréquences (KATERJI et al., $1983 b$ ) ; ce résultat qui signifierait que les $2 / 3$ de l'eau perdue par la plante provient des racines, est en bon accord avec les résultats obtenus par ailleurs (HUCK et al., 1970 ; CRUIZIAT, 1974 ; JARVIS, 1975).

G. Détermination par le calcul des durées de fonctionnement optimal de la plante : importance relative des paramètres du modèle

Afin d'illustrer l'effet des paramètres du modèle sur la cinétique de $\Psi_{F}$ et les durées de fonctionnement optimal, nous avons calculé ici la cinétique journalière de $\Psi_{F}$ pour différentes conditions pédoclimatiques caractérisées par différentes valeurs du potentiel moyen du sol $\Psi_{\mathrm{s}}$ et pour des transpirations $T$ présentant toutes une variation parabolique entre $6 \mathrm{~h}$ et $18 \mathrm{~h}$ mais avec un maximum à $12 \mathrm{~h}\left(\mathrm{~T}_{\mathrm{M}}\right)$ variable. Sur la figure 6 , n'ont été retenues que les deux conditions pédoclimatiques suivantes :

$$
\begin{aligned}
& \text { A : } \Psi_{\mathrm{S}}=-2,5 \text { bars } ; \mathrm{T}_{\mathrm{M}}=0,2 \mathrm{~mm} \cdot \mathrm{h}^{-1} \\
& \text { B : } \Psi_{\mathrm{S}}=-5,5 \text { bars } ; \mathrm{T}_{\mathrm{M}}=0,4 \mathrm{~mm} \cdot \mathrm{h}^{-1}:
\end{aligned}
$$

Par ailleurs, en ce qui concerne les 4 paramètres du modèle, on a retenu les valeurs trouvées expérimentalement sur une culture de luzerne (KATERJI et al., $1983 a$ ) ; ce sont les valeurs affectées de l'indice 0 (tabl. 1) ; mais les calculs ont été répétés pour les valeurs des paramètres inférieures ou supérieures portées sur le tableau. Enfin, la valeur critique de $\Psi_{F}$ audessous de laquelle le fonctionnement de la plante n'est plus optimal a été supposée égale à - 12 bars ; il s'agit là en effet de la valeur critique trouvée expérimentalement sur la luzerne en ce qui concerne aussi bien la résistance stomatique (KATERJI, 1982) (fig. 5) que la photosynthèse (PILARSKI, comm. pers.).

La figure 6 représente les variations journalières de $\Psi_{\mathrm{F}}$. On vérifiera que les courbes présentent une variation parabolique à l'instar des courbes $T(t)$ dans tous les cas où $\Psi_{\mathrm{F}}$ demeure supérieur au potentiel critique $\Psi_{\mathrm{pc}}$ (non intervention de la réserve plante, validité de la formule $\Psi_{\mathrm{F}}=\Psi_{\mathrm{S}}-\mathrm{RT}$ ) ; de telles courbes correspondent aux cas où :

- $\Psi_{\mathrm{s}}$ est élevé (sol humide)

- et/ou T est faible (ciel couvert par ex.)

- et/ou $\mathrm{R}$ est faible (fort développement racinaire par ex.)

- et/ou V est nul (pas de réservoir plante)

- et/ou $\Psi_{\mathrm{pc}}$ est bas (mobilisation tardive de l'eau du réservoir-plante).

Dans les conditions inverses, telles que $\Psi_{F}$ et $\Psi_{p}$ franchissent la valeur critique de $\Psi_{\mathrm{pc}}$, la réserve intervient et la courbe offre alors une dissymétrie.

L'examen de ces courbes met en évidence l'influence des conditions pédoclimatiques et des

TABLEAU 1

Valeurs retenues des paramètres.

Values assigned for the parameters.

\begin{tabular}{ccc|crc}
\hline \hline & $\mathrm{R}\left(\mathrm{bar} . \mathrm{h} . \mathrm{mm}^{-1}\right)$ & & & \\
$\Psi_{\mathrm{s}}=-2,5$ & $\Psi_{\mathrm{s}}=-5,5$ & $\Psi_{\mathrm{s}}=-8,5$ & $\mathrm{r}\left(\mathrm{bar} . \mathrm{h} . \mathrm{mm}^{-1}\right)$ & $\left.\mathrm{V} / \rho(\mathrm{mm} . \mathrm{bar})^{-1}\right)$ & $\Psi_{\mathrm{pc}}(\mathrm{bar})$ \\
\hline $0,5 \mathrm{R}_{0}=13,25$ & $0,5 \mathrm{R}_{0}=15$ & $0,5 \mathrm{R}_{0}=25$ & $0,5 \mathrm{r}_{0}=7,5$ & & 0 \\
$\mathrm{R}_{0}=26,5$ & $\mathrm{R}_{0}=30$ & $\mathrm{R}_{0}=50$ & $\mathrm{r}_{0}=15$ & $(\mathrm{~V} / \rho)_{0}=0,14$ & $-2,5$ \\
$2 \mathrm{R}_{0}=53$ & $2 \mathrm{R}_{0}=60$ & $2 \mathrm{R}_{0}=100$ & $2 \mathrm{r}_{0}=30$ & $2(\mathrm{~V} / \rho)_{0}=0,28$ & $\left(\Psi_{\mathrm{pc}}\right)_{0}=-8,5$ \\
\hline
\end{tabular}




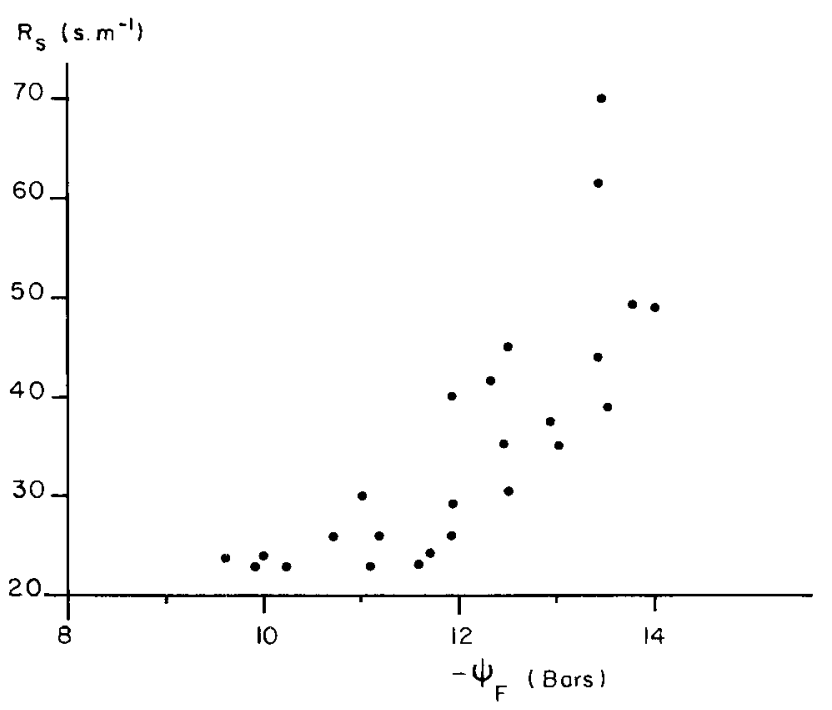

Figure 5

Relation trouvée sur une culture de luzerne entre la résistance stomatique moyenne du couvert $R_{s}$ et le potentiel hydrique foliaire $\Psi_{F}$ (d'après KATERJI, 1982).

Relationship between leaf water potential $\Psi_{F}$ and mean stomatal diffusion resistance $R_{s}$ in a lucerne crop (from KATERJ, 1982).
4 paramètres caractéristiques du modèle. Pour apprécier le poids de chacun, on notera tout d'abord que la relation de VAN DEN HONERT $\left(\Psi_{F}=\Psi_{s}-\right.$ RT) fournit, sinon la valeur exacte de $\Psi_{F}$ quand intervient la réserve, du moins un $1^{\text {er }}$ ordre de grandeur. Cela signifie que $\Psi_{\mathrm{F}}$ dépend avant tout du potentiel du sol $\Psi_{s}$, de la résistance $R$ et de la transpiration $T$. L'intervention de la réserve (qui dépend de $V / \rho$, r et $\Psi_{p c}$ ) a pour effet d'atténuer la variation de $\Psi_{F}$, de retarder l'heure ou $\Psi_{\mathrm{F}}$ atteint son minimum, et de rehausser ce minimum d'environ 3 à 4 bars (cf. courbes B-b, fig. 6).

Les courbes $\Psi_{\mathrm{F}}(\mathrm{t})$, telles que celles représentées figure 6, permettent notamment de repérer l'heure à laquelle est atteint le potentiel critique correspondant à la fermeture stomatique (valeur admise ici : - 12 bars) et d'apprécier ainsi le temps de fonctionnement optimal de la feuille. Pour préciser à cet égard l'effet des paramètres du modèle, on a répété les calculs pour d'autres conditions pédoclimatiques que celles indiquées figure 6 mais en faisant varier les 4 paramètres $\left(R, r, V / \rho\right.$ et $\left.\Psi_{p c}\right)$ de la même manière.

Les résultats obtenus publiés par ailleurs (KATERJI et al., 1983c) montrent que :
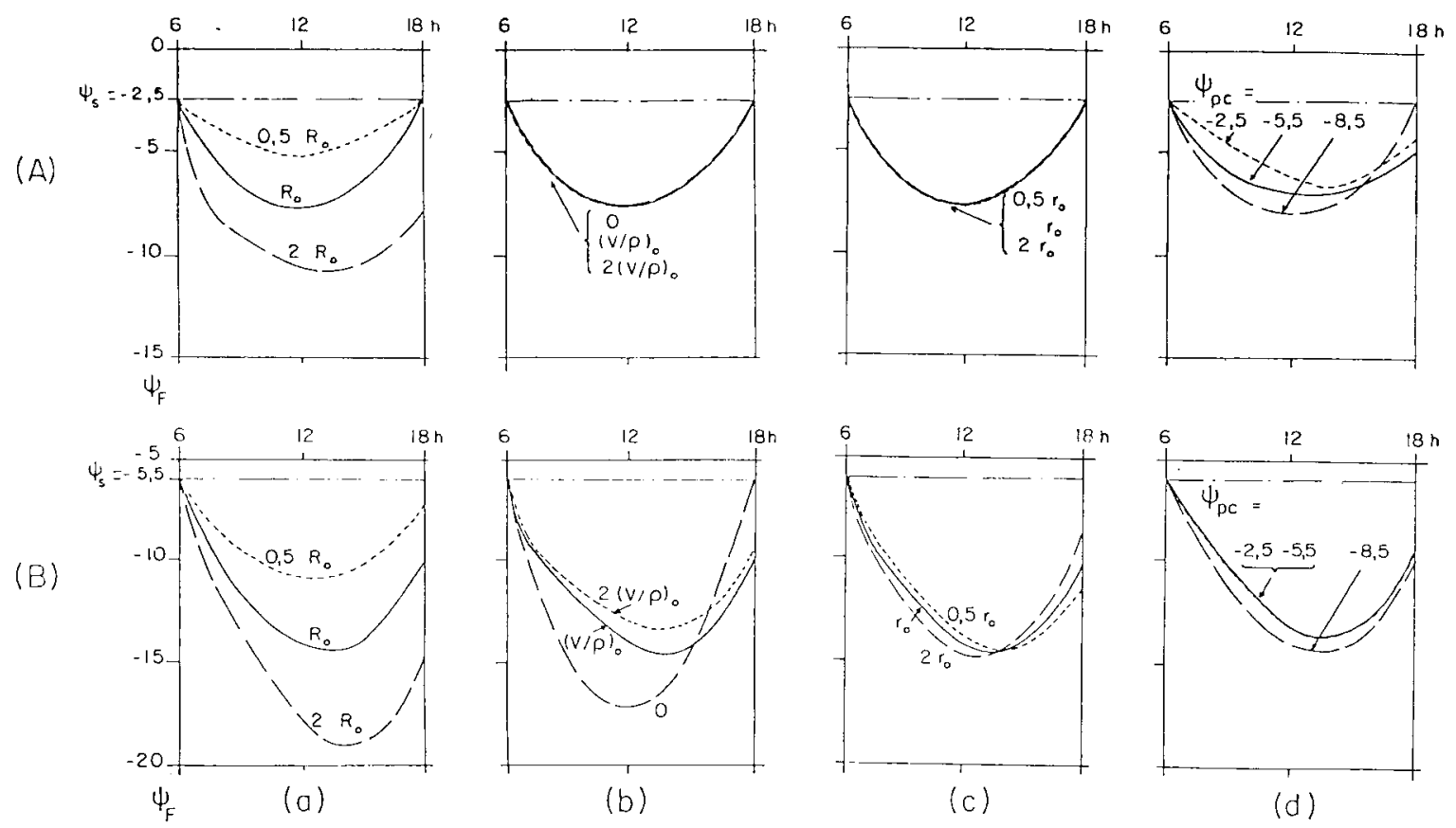

(b)

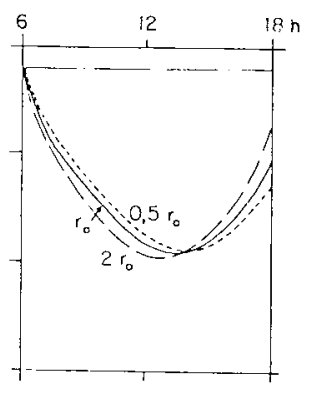

(c)

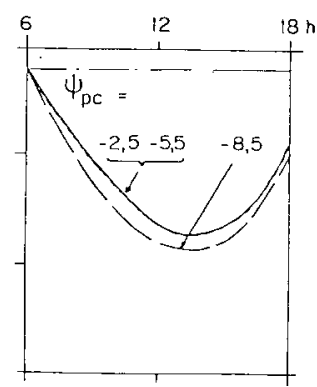

(d)

\section{Figure 6}

Courbes de variation du potentiel foliaire $\Psi_{F}$ obtenues par calcul pour 2 conditions pédoclimatiques $A$ et $B\left(A: \Psi_{S}=-2,5\right.$ bars et variation parabolique de $T$ avec $T=0$ à $6 \mathrm{~h}$ et $18 \mathrm{~h}$ et $T_{\max }=0,2 \mathrm{~mm} \cdot \mathrm{h}^{-1} \grave{a} 12 \mathrm{~h} ; \mathrm{B}: \Psi_{5}=-5,5$ bars et $T$ comme en $A$ mais avec $\left.T_{\max }=0,4 \mathrm{~mm} \cdot \mathrm{h}^{-1}\right)$.

- 3 valeurs de $R$ (graphes a) : $0,5 R_{0} ; R_{0}$ et $2 R_{0}$ avec $R_{0}=$ 26,5 bars. h. $\mathrm{mm}^{-1}$ pour $\Psi_{s}=-2,5$ bars et $R_{0}=30$ bar. h. $\mathrm{mm}^{-1}$ pour $\Psi_{s}=-5,5$ bars.

- 3 valeurs de $V / o$ (graphes b) : $0,(V / \rho)_{0}=0,14 \mathrm{~mm} . \mathrm{bar}^{-1}$ et $2(V / \rho)_{0}$

-3 valeurs de $r$ (graphes c) :0,5 $r_{0} r_{0}=15$ bar. h. $\mathrm{mm}^{-1}$ et $2 r_{0}$. -3 valeurs de $\Psi_{p c}$ (graphes $\left.d\right):-2,5 ;-5,5$ et $\left(\Psi_{p c}\right)_{0}=-8,5$ bars (pour chaque figure, les autres paramètres gardent la valeur nominale : $R_{0},(V / \rho)_{0}, r_{0},\left(\Psi_{p c}\right)_{0}$.
Graphs obtained by calculation of the variation in leaf potential $\psi_{F}$ for 2 pedoclimatic conditions $A$ and $B\left(A: \Psi_{s}=-2.5\right.$ bar and parabolic variation in $T$ with $T=0$ at 06.00 and 18.00 and $T_{\max }=0.2 \mathrm{~mm} \cdot \mathrm{h}^{-I}$ at $12.00 ; B: \Psi_{s}=-5.5 \mathrm{bar}$ and $T$ as in $A$ but with $T_{\max }=0.4 \mathrm{~mm} . \mathrm{h}^{-1}$ );

a) 3 values of $R: 0.5 R_{0} ; R_{0}$ and $2 T_{0}$ with $R_{0}=26.5 \mathrm{bar} . \mathrm{h} . \mathrm{mm}^{-1}$ for $\Psi_{s}=-2.5$ bar and $R_{0}=30$ bar.h.mm ${ }^{-1}$ for $\Psi_{s}=-5.5 \mathrm{bar}$; b) 3 values of $\mathrm{V} / \rho: 0,(\mathrm{~V} / \rho)_{0}=0.14 \mathrm{~mm}$. bar ${ }^{-1}$ and $2(\mathrm{~V} / \rho)_{0}$;

c) 3 values of $r: 0.5 r_{0}, r_{0}=15$ bar. h. $\mathrm{mm}^{-1}$ and $2 r_{0}$;

d) 3 values of $\Psi_{p c}$ : $-2.5 ;-5.5$ and $\left(\Psi_{p c}\right)_{0}=-8.5$ bar. (For each figure, the other parameters keep their "initial" value: $R_{0}$, $(V / \rho)_{0}, r_{0},\left(\Psi_{p c}\right)_{0}$. 
- dans les conditions sévères caractérisées par un $\Psi_{\mathrm{s}}$ bas (sol sec), R et $\mathrm{T}$ élevés telles que la valeur critique de $\Psi_{\mathrm{s}}$ est atteinte de toute façon relativement tôt dans la matinée, les paramètres de la réserve $(V / \rho, r$ et $\Psi_{\mathrm{pc}}$ ) interviennent en prolongeant la durée de fonctionnement optimal de 0,7 à $2 \mathrm{~h}$. Bien que limitée, cette action est loin d'être négligeable ;

- en conditions moins sévères ( $\Psi_{s}$ plus élevé, $T$ et R plus bas), les effets de $V / \rho$, r et $\Psi_{p c}$ peuvent apparaître encore plus importants puisque, selon leurs valeurs, le fonctionnement optimal de la plante peut être limité aux 2 ou $3 \mathrm{~h}$ du début de la matinée ou intéresser la journée toute entière.

En définitive, la fonction de réservoir jouée par la plante au cours du cycle diurne n'introduit qu'un correctif de 3 ou 4 bars maximum sur la cinétique diurne de $\Psi_{\mathrm{F}}$ mais ce correctif peut avoir un effet important sur la durée de fonctionnement optimal de la plante.

\section{CONCLUSION : CONSÉQUENCES SUR LE CHOIX DES GRANDEURS DE RÉFÉRENCE}

Dans l'étude des actions de l'alimentation hydrique sur la biologie de la plante, il y a lieu de distinguer :

- les grandeurs (X) exprimant l'état hydrique du végétal,

- les grandeurs $(Y)$ exprimant les conditions d'alimentation hydrique.

La grandeur (X) qu'il semble le plus intéressant de retenir est le potentiel foliaire $\Psi_{\mathrm{F}}$.

- Les grandeurs $(\mathrm{Y})$ à considérer sont celles qui déterminent $\Psi_{\mathrm{F}}$ à tout instant. Par ordre d'importance décroissante, ce sont :
- Le potentiel moyen du sol dans la zone racinaire $\overline{\Psi_{s}}$, lequel se confond normalement avec le potentiel foliaire d'équilibre en fin de nuit ou potentiel de base $\left(\Psi_{\mathrm{Fb}}\right)$. Sa valeur dépend à la fois de l'état de dessèchement du sol et de l'ensemble densité et profondeur du système racinaire.

- L'évapotranspiration réelle instantanée $E T R$ et la résistance $(R) \mathrm{du}$ circuit principal sol-feuille puisqu'en $1^{\text {re }}$ approximation (et du moins pour des cultures couvrantes pour lesquelles $T$ est très voisine de ETR) le produit R.ETR exprime la chute de potentiel foliaire par rapport à $\Psi_{s}$. $R$ dépend dans une certaine mesure de l'humidité du sol, mais surtout de la densité du système racinaire.

- Les 3 paramètres du modèle $V / \rho, \Psi_{p c}$ et $r$ qui déterminent la participation du réservoir-plante, puisque cette dernière peut avoir pour effet de diminuer de 3 à 4 bars l'amplitude de $\Psi_{\mathrm{F}}$ et d'accroître ainsi de plusieurs heures la durée de fonctionnement optimal de la plante.

L'ensemble des paramètres de type $\mathrm{Y}$ et, en particulier, les 4 paramètres du modèle proposé $(R, r, V / \rho$ et $\Psi_{\mathrm{pc}}$ ), peuvent être utilisés pour le calcul systématique de la cinétique de $\Psi_{\mathrm{F}}$ et, par là même, pour l'interprétation du fonctionnement du végétal. Mais on pourra aussi et plus simplement retenir que, commandant le fonctionnement du végétal par le biais de $\Psi_{\mathrm{F}}$, ils méritent d'être considérés comme des critères d'adaptation et de sélection et d'être mesurés, analysés et comparès en tant que tels.
Reçu le 22 décembre 1983. Accepté le 26 juin 1984.

\section{RÉFÉRENCES BIBLIOGRAPHIQUES}

Barrs H. D., 1971. Cyclic variations in stomatal aperture, transpiration and leaf water potential under constant environmental conditions. Annu. Rev. Plant Physiol., 22, 223-236.

Bavel Van C. H. M., Ahmed J., 1976. Dynamic simulation of water depletion in the root zone. Ecol. Model., 2, 189-212.

Begg J. E., Turner N. C., 1976. Crop water deficits. $A d v$. in Agron., 28, 161-217.

Berger A., 1970. Le potentiel hydrique et la résistance à la diffusion dans les stomates indicateurs de l'état hydrique de la plante, 201212. In : "Réponse des plantes aux facteurs climatiques", Coll. Uppsala (Suède), UNESCO Paris, $574 \mathrm{p}$

Berger A., 1971. La circulation de l'eau dans le système sol-plante. Etude de quelques résistances en relation avec certains facteurs du milieu. Thèse Doct. Etat, Univ. Montpellier, 224 p.

Bouchet R. J., Robelin M., 1969. Evapotranspiration potentielle et réelle. Domaine d'utilisation. Portée pratique. Bull. Tech. Inf. Min. Agric., 238, 215-223.

Boyer J. S., 1971. Nonstomatal inhibition of photosynthesis in sunflower at low leaf water potentials and high light intensities. Plant Physiol., 48, 532-536.

Boyer J. S., 1976. Photosynthesis at low water potentials. Phil. Trans. R. Soc. London, B, 273, 501-512.

Brix H., 1962. The effect of water stress on the rates of photosynthesis and respiration in tomato plants and loblolly pine seedlings. Physiol. Plant, 15, 10-20.

Buriol G. A., 1981. Etude de l'évolution de la masse d'eau des feuilles grâce à l'utilisation d'une méthode non destructive. Thèse Doct. Ing., Univ. Paris VII, 94 p.
Cowan I. R., 1972. Oscillations in stomatal conductance and plant functioning associated with stomatal conductance : observation and a model. Planta, 106, 185-219.

Cruiziat P., 1974. Détermination des pertes en eau subies par les différents organes d'une plante soumise au dessèchement. Ann. agron., 4, 539-554.

Cruiziat P., 1978. La circulation de l'eau dans la plante en flux non conservatif : quelques faits et problèmes. La Houille Blanche, 3/4, 243-253.

Cruiziat P., Thomas D. A., Bodet C., 1979. Comparaison entre mesures locales et mesure globale de résistance stomatique de feuilles de tournesol (Helianthus annuus). Oecol. Plant., 14, 447-459.

Graziani Y., Livne A., 1971. Deshydratation, water fluxes and permeability of tobacco leaf tissue. Plant Physiol., 48, 575-579.

Hallaire M., 1964. Le potentiel efficace de l'eau dans le sol en régime de dessèchement, 27-62. In : "L'eau et la production végétale ", I.N.R.A. Paris éd.

Hansen G. K., 1971. Photosynthesis, transpiration and diffusion resistance in relation to water potential in leaves during water stress. Acta Agric. Scand., 21, 163-171.

Honert T. H. Van den, 1948. Water transport in plants as catenary process. Faraday Soc., Discuss. 3, 146-153.

Hsiao T. C., Acevedo E., Fereres E., Henderson D. W., 1976. Stress metabolism, water stress growth and osmotic adjustment. Phil. Trans. R. Soc. Lond., B, 273, 479-500.

Huck M. G., Klepper B., Taylor H. M., 1970. Diurnal variations in root diameter. Plant Physiol., 45, 529-530. Int. Soc. Soil Sci., 1974, Soil physics terminol., I.S.S.S., Bull. 44, 10-17. 
Itier B., 1981. Une méthode simple pour la mesure de l'évapotranspiration réelle à l'échelle de la parcelle. Agronomie, 1, 869-876.

Jarvis P. G., 1975. Water transfer in plants. In : D. A. de Vries, N. H. Afgan : « Heat and mass transfer in the biosphere », J. Wiley $\&$ sons New York, $594 \mathrm{p}$.

Jordan W. R., Ritchie J. T., 1971. Influence of soil water stress on evaporation root absorption and internal water status of cotton. Plant Physiol., 48, 783-788.

Kanemasu E. T., Tanner C. B., 1969. Stomatal diffusion resistance of snap beans. Effect of light. Plant Physiol., 44, 1542-1546.

Katerji N., 1982. Etude et modélisation des transferts hydriques dans le système sol-plante-atmosphère. Thèse d'état, Univ. Paris VII, $197 \mathrm{p}$. + annexe $97 \mathrm{p}$.

Katerji N., Hallaire M., Perrier A., Durand R., 1983a. Transfert hydrique dans le végétal. I. Modélisation à l'échelle du couvert végétal en conditions naturelles. Acta Oecol., Oecol. Plant, 4, 11-26. 26.

Katerji N., Hallaire M., Menoux-Boyer Y., Perrier A., $1983 b$. Transfert hydrique dans le végétal. II. Détermination au champ des variations de contenu en eau du couvert végétal. Acta Oecol., Oecol. Plant., 4, 171-181.

Katerji N., Perrier A., Oulid Aissa A. K., 1983c. Exploration au champ et interprétation de la variation horizontale et verticale de la résistance stomatique: cas d'une culture de luzerne (Medicago sativa L.). Agronomie, 3, 847-856.

Katerji N., Hallaire M., Durand R., 1984. Transfert hydrique dans le végétal. III. Simulation du rôle des paramètres caractéristiques du couvert sur l'évolution au cours de la journée du potentiel hydrique foliaire. Acta Oecol., Oecol. Plant., 5 (2), 107-117.

Klepper B., 1968. Diurnal pattern of water potential in woody plants. Plant Physiol., 43, 1931-1934.

Ludlow M. M., 1976. Ecophysiology of C4 grasses, 364-386 ; In : Lange O. L. et al. : «Water and plant life », Springer Verlag, Berlin, $536 \mathrm{p}$.

Ludlow M. M., Ng T. T., 1976. Effect of water deficit on carbon dioxide exchange and leaf elongation rate of Panicum maximum var. trichoglume. Aust. J. Plant Physiol., 3, 401-413.

McPherson H. G., Slatyer R. O., 1973. Mechanisms regulating photosynthesis in Pennisetum typhaides. Aust. J. Biol. Sci., 26, 329-339.

Maertens C., 1981. Etude du rôle de l'enracinement dans les transferts d'eau du sol vers l'atmosphère. C.R. de fin d'étude d'une recherche financée par la DGRST, Station d'Agronomie de Toulouse, $30 \mathrm{p}$.

Mederski H. J., Chen L. H., Curry R. B., 1975. Effect of leaf water deficit on stomatal and non stomatal regulation of net $\mathrm{CO}_{2}$ assimilation. Plant Physiol,, 55, 589-593.

Moldau K. H., 1972. Effects of a water deficit and the light regime on photosynthetic activity of leaves. Sov. Plant Physiol., 19, 970975 .
Monteith J. L., 1965. Evaporation and environment, 205-234. In : Fogg G. E. : " The state and movement of water in living organisms », Cambridge Univ. Press.

Perrier A., 1975. Etude de l'évapotranspiration dans les conditions naturelles. III. Evapotranspiration réelle et potentielle des couverts végétaux. Ann. Agron., 26, 229-243.

Perrier A., Itier B., Bertolini J. M., Katerji N. B., 1976. A new device for continuous recording of the energy balance of natural surface. Agric. Meteorol., 16, 71-84.

Picot P., 1983. Contribution à l'étude du comportement hydrique de descendants issus du croisement Helianthus argophyllus avec Helianthus annuus. Mémoire de fin d'étude, Inst. Sup. Agric. Beauvais, $72 \mathrm{p}$.

Puech J., Marty J. R., Maertens C., 1976. Valorisation de l'eau consommée par les cultures en présence ou non d'irrigation. Bull. Tech. Inf. Min. Agric., 307, 99-103.

Reiscosky D. C., Kaspar T. C., Taylor H. M., 1982. Diurnal relationship between evapotranspiration and leaf water potential of field-grown soybeans. Agron. J., 74, 667-673.

Roy J., 1980. Comportement photosynthétique et hydrique de la feuille chez Dactylis glomerata $L$. Adaptation phénotypique et génotypique à la sécheresse. Thèse $3^{\mathrm{e}}$ cycle, Univ. Montpellier, $118 \mathrm{p}$. + annexe $153 \mathrm{p}$.

Saugier B., 1974. Transport de $\mathrm{CO}_{2}$ et de vapeur d'eau à l'interface végétation-atmosphère. Interaction du microclimat avec le comportement physiologique de plantes prairiales. Thèse Doct. d'état, Univ. Montpellier, 155 p. + annexe.

Shimshi D., 1963. Effect of soil moisture and phenylmercuric acetate upon stomatal aperture, transpiration and photosynthesis. Plant Physiol., 38, 713-721.

Slatyer R. O., 1973. Effects of short periods of water stress on leaf photosynthesis. In : "Symposium on plant response to climatic factors » Slatyer R. O. ed. UNESCO, Proc. of the Uppsala Symposium, $574 \mathrm{p}$.

Steinhardt R., Ehlers W., Van der Ploeg R. R., 1981. Analysis of soil water uptake from a drying loess soil by an oat crop using a simulation model. Irrig. Sci., 2, 237-258.

Sucoff E., 1972. Water potential in red pine : soil moisture, evapotranspiration, crown position. Ecology, 53, 681-686.

Troughton J. H., 1969. Plant water status and carbon dioxide exchange of cotton leaves. Aust. J. Biol. Sci., 22, 289-302.

Turner N. C., 1969. Stomatal resistance to transpiration in three controlling canopies. Crop Sci., 9, 303-307.

Waring R. H., 1969. Forest plants of the eastern siskiyous : their environmental and vegetational distribution. Northwest Sci., 43, 117.

Willis A. J., Balasubramaniam S., 1968. Stomatal behavior in relation to rates of photosynthesis and transpiration in Pelargonium. New Phytol., 67, 265-285. 